\title{
A practical guide and power analysis for GLMMs: Detecting among treatment variation in random effects
}

Morgan P Kain, Benjamin M Bolker, Michael W McCoy

In ecology and evolution GLMMs are becoming increasingly used to test for differences in variation by treatment at multiple hierarchical levels. Yet, the specific sampling schemes that optimize the power of an experiment to detect differences in random effects by treatment/group remain unknown. In this paper we develop a blueprint for conducting power analyses for GLMMs focusing on detecting differences in variance by treatment. We present parameterization and power analyses for random-intercepts and random-slopes GLMMs because of their generality as focal parameters for most applications and because of their immediate applicability to emerging questions in the field of behavioral ecology. We focus on the extreme case of hierarchically structured binomial data, though the framework presented here generalizes easily to any error distribution model. First, we determine the optimal ratio of individuals to repeated measures within individuals that maximizes power to detect differences by treatment in among-individual variation in intercept, among-individual variation in slope, and within-individual variation in intercept.

Second, we explore how power to detect differences in target variance parameters is affected by total variation. Our results indicate heterogeneity in power across ratios of individuals to repeated measures with an optimal ratio determined by both the target variance parameter and total sample size. Additionally, power to detect each variance parameter was low overall (in most cases $>1,000$ total observations per treatment needed to achieve $80 \%$ power) and decreased with increasing variance in non-target random effects. With growing interest in variance as the parameter of inquiry, these power analyses provide a crucial component for designing experiments focused on detecting differences in variance. We hope to inspire novel experimental designs in ecology and evolution investigating the causes and implications of individual-level phenotypic variance, such as the adaptive significance of within-individual variation. 
Title: A practical guide and power analysis for GLMMs: Detecting among treatment variation in random effects

Authors: Morgan P. Kain ${ }^{1 *}$, Ben M. Bolker², Michael W. McCoy ${ }^{1}$

${ }^{1}$ Department of Biology East Carolina University, N108 Howell Science Complex, Greenville, NC 27858

${ }^{2}$ Department of Mathematics \& Statistics, McMaster University, 314 Hamilton Hall, 1280 Main

Street West, Hamilton, Ontario L8S 4K1, Canada

* Correspondence author.

N108 Howell Science Complex

East Carolina University

Mail Stop 551

Greenville, NC 27858

E-mail: morganpkain@gmail.com

\begin{abstract}
In ecology and evolution GLMMs are becoming increasingly used to test for differences in variation by treatment at multiple hierarchical levels. Yet, the specific sampling schemes that optimize the power of an experiment to detect differences in random effects by treatment/group remain unknown. In this paper we develop a blueprint for conducting power analyses for GLMMs focusing on detecting differences in variance by treatment. We present parameterization and power analyses for random-intercepts and random-slopes GLMMs because of their generality as focal parameters for most applications and because of their immediate applicability to emerging questions in the field of behavioral ecology. We focus on the extreme case of hierarchically structured binomial data, though the framework presented here generalizes easily to any error distribution model. First, we determine the optimal ratio of individuals to repeated measures within individuals that maximizes power to detect differences by treatment in amongindividual variation in intercept, among-individual variation in slope, and within-individual variation in intercept. Second, we explore how power to detect differences in target variance parameters is affected by total variation. Our results indicate heterogeneity in power across ratios of individuals to repeated measures with an optimal ratio determined by both the target variance parameter and total sample size. Additionally, power to detect each variance parameter was low overall (in most cases $>1,000$ total observations per treatment needed to achieve $80 \%$ power) and decreased with increasing variance in non-target random effects. With growing interest in variance as the parameter of inquiry, these power analyses provide a crucial component for designing experiments focused on detecting differences in variance. We hope to inspire novel experimental designs in ecology and evolution investigating the causes and implications of individual-level phenotypic variance, such as the adaptive significance of within-individual variation.
\end{abstract}

Key-words: individual variation, behavioral ecology, reaction norm, plasticity, binomial distribution, hierarchical, sampling scheme 


\section{Introduction}

2 Recent advances in computing power and access to increasingly sophisticated statistical

3 tools such as generalized linear mixed effects models are changing research in ecology, evolution

4 and behavior. Research questions and data analyses are no longer confined to the assumptions of

5 clean experimental designs based on agricultural plots and Normal error distributions.

6 Researchers now commonly incorporate multiple levels of hierarchical nesting (e.g. repeated

7 measures) and can analyze data using a wide array of non-Gaussian error distribution models.

8 This change is epitomized by the recent increase in use of linear and generalized linear mixed

9 models ([G]LMMs: Bolker et al., 2009; Touchon, J. \& McCoy, W.M. unpublished data). These

10 powerful tools permit appropriate modeling of variation among groups and across space and

11 time, allowing for more accurate extrapolation of statistical results to unobserved data, as well as

12 statistical tests of variance components (Gelman \& Hill, 2006; Bolker et al., 2009; Zuur et al.,

13 2009; Zuur, Hilbe \& Leno, 2013).

14 The upsurge in the use of LMM and GLMM has been facilitated by several recent methods

15 papers (Bolker et al., 2009; Martin et al., 2011; Dingemanse \& Dochtermann, 2013; Schielzeth

16 \& Nakagawa, 2013) and textbooks (Gelman \& Hill, 2006; Zuur et al., 2009; Zuur, Hilbe \& Leno,

17 2013; Bolker, 2015) specifically aimed at non-statisticians. While these resources have

18 accelerated the adoption of these tools, there are still too few resources guiding researchers

19 through the choices that must be made prior to the initiation of a new experiment, such as the

20 sampling scheme that will optimize the power of an experiment requiring analysis by linear

21 (Moineddin, Matheson \& Glazier, 2007; Scherbaum \& Ferreter, 2009; Martin et al., 2011) and

22 generalized linear (Johnson et al., 2014) mixed models. In this paper, we develop a blueprint for

23 conducting power analyses for GLMMs using the lme 4 package (Bates et al., 2014) in the R 
24 statistical programming environment (R Development Core Team, 2015). We focus on a specific 25 application aimed at detecting differences in variance among- and within-groups between

26 clusters of groups, such as differences in the amount of variation among individuals (group)

27 between the treatments (cluster) of a manipulative or observational experiment.

Power analysis is fundamental to good experimental design, but is often overlooked

(Jennions \& Moller, 2003), or in the case of GLMMs, simply too difficult to implement for many practitioners. Power analyses can be especially daunting for GLMMs because they require large simulations with complex, non-Normal and non-independent data structures (Johnson et al., 2014). In this paper we take advantage of recent developments in the $l$ me 4 package in $R$ that simplify the process of simulating appropriate data (>version 1.1-6). Despite the increasing use of GLMMs in ecology and evolution and growing interest in variance, we are aware of no papers that present power analyses for statistical tests on variance using GLMMs, and only one paper presenting power analyses for fixed effects in GLMMs (Johnson et al., 2014). Indeed, Johnson et al.'s (2014) analysis illustrates that power analyses conducted for hierarchically structured experiments that do not incorporate random effects can generate biased estimates of fixed effects, highlighting the need for a better understanding of these approaches. While most applications of GLMMs to date have focused on detecting differences in fixed effects while appropriately accounting for random effects (e.g. Johnson et al., 2014), GLMMs are under rapid development and many new applications are now possible (e.g. modeling heterogeneous error variance: Kizilkaya \& Tempelman 2005, Cernicchiaro et al., 2013). With growing interest in variance as the parameter of inquiry (Moore, Brodie \& Wolf, 1997; Lynch \& Walsh, 1998; Benedetti-Cecchi, 2003; Hill \& Zhang, 2004; Nussey, Wilson \& Brommer, 2007; Dingemanse et al., 2010; Tonsor, Elnaccash \& Scheiner, 2013; Westneat, Wright \& Dingemanse, 
47 2014), there is an increased need for accessible, flexible simulation-based power analyses that

48 assess power to detect differences in random effects - the magnitude of variation present among

49 repeated measures at a specific hierarchical level (Gelman \& Hill, 2006; Zuur et al., 2009)—by

50 treatment.

51 Here we present parameterization and power analyses for random-intercepts and random-

52 slopes GLMMs that test for differences in variation among- and within-groups (e.g. differences

53 in the amount of variation among- and within-individuals in different treatments of an

54 experiment). We focus on three key parameters: 1) Among-group variation in intercept; 2)

55 Within-group variation in intercept; 3) Among-group variation in slope. We examine each of

56 these comparisons in two contexts. First, we describe the optimal ratio of groups (e.g. hospitals,

57 schools or individuals) to observations within groups (e.g. patients, students, repeated

58 observations of each individual) that maximizes power to detect differences in each variance

59 parameter. In experiments with binomially distributed response variables, observations within

60 groups are organized into $j$ sampling occasions, each containing $n$ Bernoulli observations (e.g.

61 individuals are each measured $n$ times for the presence or absence of a behavior in each sampling

62 occasion $j$ ). Here we discuss the ratio of groups to total observations within groups ( $n * j)$, and

63 consider how varying $n$ and $j$ affect power to detect each variance parameter. Second, we explore

64 how power to detect differences in specific variance parameters is affected by increasing

65 variation in non-target parameters (e.g., how power to detect differences in among-group

66 variation decreases as within-group variance increases). We consider both random-intercepts and

67 random-slopes models because of their generality as focal parameters for most applications, and

68 choose to focus on the extreme case of hierarchically structured binomial data because binary

69 response data (e.g. the presence or absence of a behavior) contains the least possible amount of 
70 information per observation and yet is a common data format for a variety of endpoints measured 71 in ecology.

72 We use vocabulary and examples from behavioral ecology to illustrate our models because

73 of their immediate applicability to emerging questions in this field. Specifically, we evaluate

74 power to detect significant differences in among-individual variation in reaction norm intercept

75 and slope, and within-individual variation in intercept between individuals (i.e. among

76 individuals aggregated by treatment) (Nussey, Wilson \& Brommer, 2007; Dingemanse et al.,

77 2010). Our methods extend current approaches used in behavioral ecology for quantifying

78 among-individual variation away from simply testing whether there is significant deviation from

79 a null model of no variation (Martin et al., 2011; Van de Pol et al., 2012; Dingemanse \&

80 Dochtermann, 2013) toward quantifying and contrasting the magnitude of among- and within-

81 individual variation among multiple groups of individuals.

82 While we focus on behavioral ecology as the primary application for these power analyses,

83 these analyses are generally appropriate for comparing variation in hierarchically structured data.

84 For example, similar methods could be used to evaluate power to detect the effects of a new

85 experimental district-wide policy on variation among schools in student performance, or to

86 evaluate variation among individuals in foraging success between populations (e.g. birds in an

87 urban environment experience canalized behavior relative to birds in a natural environment, 88 possibly reducing diversification; see Fernando De Leon et al., 2011).

89 In an effort to present a framework that is customizable for a diversity of research

90 problems, we focus on a general sampling scheme in which several Bernoulli observations ( $n>$

91 1) within multiple sampling occasions $(j>1)$ are available for each individual. Under this

92 sampling scheme multiple probabilities of "success" (e.g. the probability of displaying a 
93 behavior) are available for each individual, which is necessary for quantifying within-individual

94 variation (variation in the probability an individual displays a behavior between sampling

95 occasions). However, we note that often in behavioral ecology only a single Bernoulli

96 observation $(n=1)$ is available for each sampling occasion $j$. We include a description on how to

97 modify this general case to accommodate single observations per sampling occasion in

98 Supplement 1. Finally, while we focus on the binomial GLMM, the framework presented here

99 generalizes easily to other error distribution models such as Normal, log-Normal, or Gamma (for

100 continuous responses) or Poisson or negative binomial (for count responses).

101

102 Methods

103 Linear Mixed Model

104 We begin by introducing a general linear mixed model (LMM) to illustrate the variance 105 components we are interested in (Figure 1) and their applications in behavioral ecology. We 106 provide only a brief introduction to LMMs here because they have been extensively discussed in 107 several recent reviews and textbooks (Gelman \& Hill, 2006; Zuur et al., 2009; Stroup, 2012;

108 Zuur, Hilbe \& Leno, 2013; Dingemanse \& Dochtermann, 2013; Bates et al., 2014; Bolker,

109 2015). We use the notation of Stroup (2012) to facilitate a transition to the binomial GLMM

110 model, which is the focus of our power analyses.

111 A two treatment linear mixed model can be written as:

114

115

$[1] \mathrm{y}_{\mathrm{ijk}} \mid b_{0 \mathrm{ik}}, b_{1 \mathrm{ik}} \sim \operatorname{Normal}\left(\mu_{\mathrm{ijk}}, \sigma_{\varepsilon \mathrm{kk}}^{2}\right)$

[2] $\eta_{\mathrm{ijk}}=\beta_{0 \mathrm{k}}+b_{0 \mathrm{ik}}+\left(\beta_{1 \mathrm{k}}+b_{\mathrm{ik}}\right) X_{\mathrm{ijk}}$

[3] Identity link: $\eta_{\mathrm{ijk}}=\mu_{\mathrm{ijk}}$

[4] $\left[\begin{array}{l}b_{0 \mathrm{ik}} \\ b_{1 \mathrm{ik}}\end{array}\right] \sim \operatorname{MVN}\left(\left[\begin{array}{c}0 \\ 0\end{array}\right],\left[\begin{array}{cc}\sigma^{2} 0 \mathrm{k} & \sigma_{01 \mathrm{k}} \\ \sigma_{01 \mathrm{k}} & \sigma^{2}{ }_{1 \mathrm{k}}\end{array}\right]\right)$ 

Here, a single phenotypic measurement $\mathrm{y}_{\mathrm{ijk}}$ is of individual $i$, at level $j$ of the covariate $X$

117 (in studies of animal behavior the covariate of interest is often an environmental gradient) in

118 treatment $k$. This model is composed of three components: the treatment mean in environment $j$

$119\left(\beta_{0 \mathrm{k}}+\beta_{1 \mathrm{k}} X_{\mathrm{ijk}}\right)$, the unique average response of individual $i$ across the environmental gradient $\left(b_{0 \mathrm{k}}\right.$

$\left.120+b_{1 \mathrm{k}} X_{\mathrm{ijk}}\right)$, and a residual error due to the variation around the mean of individual $i\left(\sigma_{\varepsilon \mathrm{k}}^{2}\right)$, which

121 is assumed to be homogenous across $X$ and among all individuals in treatment $k$, but is allowed

122 to vary by treatment. Individuals vary from the treatment mean reaction norm in both their

123 intercept $\left(b_{0 \mathrm{ik}}\right)$ and slope $\left(b_{1 \mathrm{ik}}\right)$, which together compose the total phenotypic variance attributable

124 to among-individual variation. This individual contribution is quantified using a random

125 intercepts and slopes model with a multivariate Normal (MVN) distribution [4]. Variation among

126 individuals in intercept and slope are $\sigma^{2}{ }_{0 \mathrm{k}}$ and $\sigma^{2}{ }_{1 \mathrm{k}}$ respectively; covariance between intercept and

127 slope is given by $\sigma_{01 k}$. In a LMM, the linear predictor directly predicts the mean, as shown by the

128 identity link function in equation [3]. In a GLMM, the linear predictor predicts a function of the

129 mean $g(x)$, which must be linearized through the use of non-identity link functions; for example,

130 we use the standard logit (log-odds) link for Binomial GLMM.

131

Among-individual variation in intercept

In behavioral ecology among-individual variation in intercept $\sigma^{2}{ }_{0 \mathrm{k}}$ describes the amount

134 of variation around average behavior that occurs among individuals (Figure 1). In field studies,

$135 \sigma^{2}{ }_{0 \mathrm{k}}$ often describes variation among individuals in their average behavior in the average

136 environment (see Nussey, Wilson \& Brommer, 2007; Westneat et al., 2011). Previous work has

137 demonstrated that individuals from a diversity of taxa vary in their average behavior in many

138 different environments (Bell, Hankison \& Laskowski, 2009). Yet, comparisons of among- and 
139 within-individual variation in average behavior (or other forms of plasticity) among populations

140 or treatments remain underrepresented (e.g. Westneat et al., 2011; Dingemanse et al., 2012). For

141 example, Westneat et al., (2011) found that female house sparrows vary less from one another in

142 their average provisioning behavior than male sparrows. In the model presented here, the random

143 intercept $\left(b_{0 \mathrm{ik}}\right)$ for each individual (e.g. male and female nest provisioning rates are drawn from

144 Normal distributions with different variances) is drawn from a treatment-specific Normal

145 distribution.

146

147 Within-Individual Variation in Intercept

148 Within-individual variation in intercept $\left(\sigma_{\varepsilon \mathrm{kk}}^{2}\right)$ is defined as the amount individuals vary

149 around their own average behavior. Within-individual variation is routinely used for the

150 calculation of repeatability in studies of animal personality (Bell, Hankison \& Laskowski, 2009;

151 Dingemanse et al., 2010) or more often is simply regarded as noise, despite the well established

152 ecological and evolutionary implications of within-individual variation (Stamps, Briffa \& Biro,

153 2012; Biro \& Adriasenssens, 2013; Westneat, Wright \& Dingemanse, 2014; Cleasby, Nakagawa

$154 \&$ Schielzeth, 2015). For example, a variable predator environment may select for individual

155 prey that vary greatly around their mean behavior to remain unpredictable (Stamps, Briffa \&

156 Biro, 2012). LMMs can directly quantify patterns of within-individual variation when repeated

157 measures within multiple individuals are available, facilitating comparisons of consistency

158 responses between individuals (Dingemanse et al., 2013). Here we are interested in determining

159 if $\sigma_{\varepsilon k}^{2}$ differs by treatment. In other words, do individuals in one population or treatment exhibit

160 more intra-individual behavioral variation than individuals from a second population or

161 treatment? 
163

164

165

166

167

Among-Individual variation in slope

Substantial empirical work has shown that individual animals in a variety of taxa display variation in phenotypic plasticity (Martin \& Réale, 2008; Mathot et al., 2011; Dingemanse et al., 2012); using mixed models to quantify this variation has been the primary focus of several recent papers (Martin et al., 2011; Van de Pol, 2012; Dingemanse and Dochtermann, 2013). Amongindividual variation in phenotypic plasticity has implications for the rate of evolutionary change, population stability and population persistence (Wolf \& Weissing, 2012; Dingemanse \& Wolf, 2013); thus defining those populations exhibiting greater individual variation in plasticity could help distinguish stable populations and populations with a high probability of micro-evolutionary change (Pigliucci, 2001; Ghalambor, Angeloni \& Carroll, 2010). To quantify group differences in plasticity variation, multiple measurements within each individual across an environmental gradient are required. Here we are interested in determining if $\sigma^{2}{ }_{1 \mathrm{k}}$ differs by treatment.

\section{Binomial GLMM}

We assess power of a binomial GLMM for detecting differences in variation by treatment. This model can be written as:

[5] $y_{\mathrm{ijk}} \mid b_{0 \mathrm{ik}}, b_{1 \mathrm{ik},} v_{\mathrm{ijk}} \sim \operatorname{Binomial}\left(N_{\mathrm{ijk}}, \pi_{\mathrm{ijk}}\right)$

$[6] \eta_{\mathrm{ijk}}=\beta_{0}+b_{0 \mathrm{ik}}+\left(\beta_{1}+b_{1 i k}\right) X_{\mathrm{ijk}}+v_{\mathrm{ijk}}$

[7] Inverse-logit: $\pi_{\mathrm{ijk}}=1 /\left(1+e^{-\eta_{\mathrm{ijk}}}\right)$

$[8]\left[\begin{array}{c}b_{0 \mathrm{ik}} \\ b_{1 \mathrm{ik}}\end{array}\right] \sim \operatorname{MVN}\left(\left[\begin{array}{l}0 \\ 0\end{array}\right],\left[\begin{array}{cc}\sigma_{0 \mathrm{k}}^{2} & \sigma_{01 \mathrm{k}} \\ \sigma_{01 \mathrm{k}} & \sigma^{2}{ }_{1 \mathrm{k}}\end{array}\right]\right)$

[9] $v_{i j k} \sim \operatorname{Normal}\left(0, \sigma^{2}{ }_{v k}\right)$ 
Here, $y_{\mathrm{ijk}}$ is the number of "successes" in $N_{\mathrm{ijk}}$ observations of the $i$ th individual in

185

186

187

188

189

190

191

192

193

194

195

196

197

198

199

200

201

202

203

204

205

206

treatment $k$ at the $j$ th sampling occasion. When an environmental covariate $(X)$ is present, we assume one sampling occasion occurs at each level of the covariate $j$. Here, in the absence of an environmental covariate, the linear predictor reduces to $\eta_{\mathrm{ijk}}=\beta_{0}+b_{0 \mathrm{ik}}+v_{\mathrm{ijk}}$ and the $j$ th occasion is simply a repeated sampling occasion in the same conditions. Note, when $N_{\mathrm{ijk}}=1$ there is only 1 observation per sampling occasion $j$, making $y_{\mathrm{ijk}}$ a Bernoulli response variable (see Supplement 1). When $y_{\mathrm{ijk}}$ is Bernoulli, overdispersion $\left(v_{\mathrm{ijk}}\right)$ and thus within-individual variation is not identifiable.

In this model $\pi_{\mathrm{ijk}}$ describes the underlying probability of individual $i$ in treatment $k$ at occasion $j$ exhibiting a behavior. Variation in $\pi$ is determined by the linear combination of predictors on the logit (log-odds) scale: group intercept $\left(\beta_{0}\right)$, group slope $\left(\beta_{1}\right)$, individual unique intercept $\left(b_{0 \mathrm{ik}}\right)$, slope $\left(b_{1 \mathrm{ik}}\right)$, and observation level overdispersion that decrease predictive power at each observation $\left(v_{\mathrm{ijk}}\right)$. This linear predictor is transformed with the inverse-logit link to produce $\pi_{\mathrm{ijk}}$, which follows a logit-Normal-binomial mixed distribution.

We use an observation-level random effect to model additive overdispersion (Browne et al., 2005), which models increased variance (following a Normal distribution with variance $\sigma^{2}{ }_{v \mathrm{k}}$ ) in the linear predictor on the link scale (Nakagawa \& Schielzeth, 2010). Overdispersion is used to quantify within-individual variation because it models variation in $\pi$ between each sampling occasion $j$ for each individual. Here the magnitude of overdispersion is allowed to vary by treatment (for an example of multiple data sets where this occurs see Hinde \& Demetrio, 2007), which is a focus of our power analysis.

The transformation through the inverse-logit function makes each of the three target variance components difficult to visualize with a concise figure. However, because the binomial 
207 GLMM model follows similar patterns as the LMM, we present power analyses for the binomial

208 GLMM using the visual aid presented for the LMM (Figure 1). Finally, we simulate data for a

209 fully balanced design without losing generality. See Martin et al., 2011 and Van de Pol, 2012 for

210 a discussion on experimental designs where individuals are assayed in partially overlapping

211 environments and when only single measurements are obtained for some individuals.

212

213 Simulations

214 All data were simulated in the R statistical programming environment using newly

215 developed simulation capabilities of the lme 4 package (>version 1.1-6, Bates et al., 2014).

216 Guidelines for parameterizing the GLMMs and running data simulations and power analyses are

217 provided in Supplement 1. For a given total sample size, we present simulations for determining

218 the optimal ratio of total number of individuals versus the number of repeated measures within

219 individuals needed to provide power to detect a difference among treatments $80 \%$ of the time.

220 We conducted simulations for multiple ratios of individuals to total observations within

221 individuals, varying both sampling occasions $(j)$ and Bernoulli observations within sampling

222 occasions $(n)$. Next, we describe simulations that evaluate how increasing "noise" (variation in

223 non-target random effects) affects power to detect differences in targeted variance comparisons.

224 For both scenarios we simulate data with biologically relevant parameter values that

225 illustrate common trends in power. At extreme parameter values the trends presented here may

226 not hold due to interactions between the variance components that arise at the boundaries of

227 binomial space. We do not dwell on these exceptions since they are unrealistic for most

228 empirical data sets, but suggest exploration of these exceptions with code provided in

229 Supplement 1. 
We ran 2800 simulations for each combination of parameter values. The significance of a

231 given random effect was assessed using likelihood ratio tests (LRTs) between models with and

232 without the focal random effect. To correct for the known conservatism of the LRT when testing

233 for $\sigma^{2}=0$ (due to a null value on the boundary of parameter space), we adopted the standard

234 correction of dividing all p-values by 2 (Pinheiro \& Bates, 2000; Verbeke \& Molenberghs, 2000;

235 Fitzmaurice, Laird \& Ware, 2004; Zuur et al., 2009). This correction was appropriate for all p-

236 values because each LRT compared models that differed in only a single degree of freedom.

237 Power is estimated as the percentage of simulations that provide a corrected p-value smaller than

2380.05 . We insured the validity of a nominal p-value of 0.05 by confirming that 2800 simulations

239 of a scenario with equivalent standard deviations in both treatments did not result in rejecting the

240 null hypothesis more than $5 \%$ of the time. Under extremely low numbers of individuals $(\sim 2-4)$

241 power to detect differences in the null case exceeded 5\% ( 10-15\%), possibly inflating power in

242 these cases. Regardless, random effects cannot be reliably estimated with such low sample sizes

243 and therefore in most cases such experimental designs should be avoided.

\section{Scenario 1: Determining the optimal sampling scheme}

246 Most researchers face limitations imposed by time, money and access to samples, and are

247 therefore confronted with the question of how resources should be divided between individuals

248 and measures within individuals. To investigate the optimal allocation of sampling effort

249 between the number of individuals and number of observations per individual, we simulated two

250 data sets for each variance comparison (See Table 1 for a summary of all simulations).

251 First, using three hypothetical total numbers of Bernoulli observations per treatment

252 (total sample size per treatment, $\mathrm{TSS}_{\mathrm{T}}$ ), we manipulated either the ratio of individuals to 
253 sampling occasions $\left(\sigma^{2}{ }_{0 \mathrm{k}}\right.$ and $\left.\sigma^{2}{ }_{1 \mathrm{k}}\right)$, or the ratio of individuals to Bernoulli observations within

254 sampling occasions $\left(\sigma^{2}{ }_{v \mathrm{k}}\right)$. For comparisons of $\sigma^{2}{ }_{0 \mathrm{k}}$ and $\sigma^{2}{ }_{1 \mathrm{k}}$ we manipulated the ratio of

255 individuals to sampling occasions, holding the number of Bernoulli observations constant at 5 ,

256 because power follows a non-monotonic pattern across these ratios for $\sigma^{2}{ }_{0 \mathrm{k}}$ and $\sigma^{2}{ }_{1 \mathrm{k}}$ (Figures 2,

257 3). Conversely, for comparisons of $\sigma^{2}{ }_{v \mathrm{k}}$ we manipulated the ratio of individuals to Bernoulli

258 observations and held the number of sampling occasions constant at 5 because power follows a

259 non-monotonic pattern across ratios of individuals to Bernoulli observations for $\sigma^{2}{ }_{v \mathrm{k}}$ (Figure 4).

260 For comparisons of $\sigma^{2} 0$, and $\sigma^{2}{ }_{v k}$ we simulated $\mathrm{TSS}_{\mathrm{T}}$ of 600,1200 and 2400 , and for

261 comparisons of $\sigma^{2}{ }_{1 \mathrm{k}} \mathrm{TSS}_{\mathrm{T}}$ were 300,600 , and 1200 . For example, for $b_{1 \mathrm{ik}}$ with a $\mathrm{TSS}_{\mathrm{T}}$ of 300 , the

262 most extreme ratios were 30 individuals with 2 sampling occasions and 2 individuals with 30

263 sampling occasions. While using only 2 samples for a grouping variable (individuals) is never

264 suggested for a random effect, we include this combination as an illustration of the low power

265 that results from an ill-conceived sampling scheme. For each variance comparison we simulated

266 three different effect sizes (2, 2.5, and 3 fold difference in standard deviation by treatment).

267 Next, we simulated data sets with increasing numbers of Bernoulli observations for

268 comparisons of $\sigma^{2}{ }_{0 \mathrm{k}}$ and $\sigma^{2}{ }_{1 \mathrm{k}}$ (Figure 5A, B) and with increasing numbers of sampling occasions

269 for comparisons of $\sigma^{2}{ }_{v \mathrm{k}}$ (Figure 5C). For these simulations we used 1, 3, 5, 10 and 15 Bernoulli

270 observations or sampling occasions. Ratios of individuals to sampling occasions $\left(\sigma^{2}{ }_{0 \mathrm{k}}\right.$ and $\left.\sigma^{2}{ }_{\mathrm{lk}}\right)$

271 or individuals to Bernoulli observations $\left(\sigma^{2}{ }_{v k}\right)$ followed the intermediate $\mathrm{TSS}_{\mathrm{T}}$ from the

272 simulations described above. For example, for comparisons of $\sigma^{2}{ }_{0 \mathrm{k}}$ we simulated $1,3,5,10$ and

27315 Bernoulli observations for ratios of individuals to sampling occasions ranging from 120:2 to

274 2:120. For all comparisons we simulated data using an effect size of a 2.5 fold difference in

275 standard deviation by treatment. 

treatments. For comparisons of among-individual variation in intercept no environmental covariate was used causing each sampling occasion to occur in the same conditions.

279 Additionally, $\sigma_{v \mathrm{k}}^{2}$ was held constant among treatments. For comparisons of among-individual variation in slope we held $\sigma^{2}{ }_{v \mathrm{k}}$ constant. Finally, for comparisons of within-individual variation

in intercept, no environmental covariate was included and $\sigma^{2}{ }_{0 \mathrm{k}}$ was held constant among treatments. All parameter values used in simulations for both Scenarios can be found in Table S1.

Our goal in Scenario 1 was to isolate changes in a single variance parameter, but exploration of the dependence among multiple variance components and the mean may be warranted if it is relevant for a specific problem. Incorporating concurrent changes in intercept, code presented in the online supplement. We show initial results of relaxing some of these assumptions in Scenario 2, but full exploration of these possibilities are beyond the scope of this paper.

291

\section{Scenario 2: Measuring the ratio of overdispersion to effect size}

294 influences power to detect differences in the target variance among treatments. Therefore, we 295 simulated four levels of "noise" (magnitude of non-target random effect variance) assuming a 296 Normal distribution with increasing standard deviations $(0.1,0.5,1.0,2.0)$ (Figure 6). These 297 correspond to ratios of target variance parameter effect size to non-target variance of $25: 1,5: 1$, 298 5:2, and 5:4. For comparisons of $\sigma^{2}{ }_{0 \mathrm{k}}$ and $\sigma^{2}{ }_{1 \mathrm{k}}$ " "noise" was simulated with increasing variation in 
299 within-individual variation $\left(\sigma^{2}{ }_{v \mathrm{k}}\right)$, while for comparisons of $\sigma^{2}{ }_{v \mathrm{k}}$ noise was simulated with

300 among-individual variation in intercept $\left(\sigma^{2}{ }_{0 \mathrm{k}}\right)$. For each variance parameter ratios of individuals

301 to repeated measures followed the largest $\mathrm{TSS}_{\mathrm{T}}$ sampling scheme used in Scenario 1 and an ES

302 of a $2.5 x$ difference in standard deviation by treatment.

303

304 Results

305 Scenario 1: Determining the optimal sampling scheme

306 Power to detect differences between treatments for each variance component increases

307 with total sample size $\left(\mathrm{TSS}_{\mathrm{T}}\right)$ and effect size (ES) (Figures 2-5). For a given $\mathrm{TSS}_{\mathrm{T}}$ power

308 depends on the ratio of the number of individuals to the number of repeated measures per

309 individual; however, the optimal ratio of individuals to repeated measures varies depending on

$310 \mathrm{TSS}_{\mathrm{T}}$ and target variance parameter. For example, power to detect both $\sigma^{2}{ }_{0 \mathrm{k}}$ and $\sigma^{2}{ }_{1 \mathrm{k}}$ is non-

311 monotonic across ratios of individuals to sampling occasions (Figures 2, 3), but is an increasing

312 function of the number of Bernoulli observations within sampling occasions (Figure 5A, B).

313 Additionally, for each variance parameter the ratio of individuals to repeated measures within

314 individuals that maximizes power is dependent on both $\mathrm{TSS}_{\mathrm{T}}$ and ES. As $\mathrm{TSS}_{\mathrm{T}}$ and ES increases,

315 greater numbers of individuals relative to repeated measures within individuals leads to higher

316 power for each variance parameter.

At a low sample size $\left(\mathrm{TSS}_{\mathrm{T}}=600\right)($ Figure $2 \mathrm{~A})$ power to detect $\sigma^{2}{ }_{0 \mathrm{k}}$ is maximized at a

318 ratio of individuals to sampling occasions of $6: 5$ at smaller effect sizes $(2 \mathrm{x}, 2.5 \mathrm{x})$ and 10:3 at a

319 large effect size (3x). Under a larger sample size and a small effect size $\left(\mathrm{TSS}_{\mathrm{T}}=2400, \mathrm{ES}=2 \mathrm{x}\right)$

320 (Figure 2C) power is maximized at a ratio of approximately 2:1, while under a large sample size 
321 and large effect size $\left(\mathrm{TSS}_{\mathrm{T}}=2400, \mathrm{ES}=2.5 \mathrm{x}, 3 \mathrm{x}\right)($ Figure $2 \mathrm{C})$, power is maximized at ratios

322 ranging from approximately 5:1 to $13: 1$.

323 At a low sample size and effect size $\left(\mathrm{TSS}_{\mathrm{T}}=300, \mathrm{ES}=2 \mathrm{x}\right)$, power to $\operatorname{detect} \sigma^{2}{ }_{1 \mathrm{k}}$ is

324 maximized at a ratio of 12:5 (Figure 3A), while larger sample sizes and effect sizes (e.g. TSS $_{\mathrm{T}}=$

$\left.325600, \mathrm{ES}=2.5 \mathrm{x}, 3.0 \mathrm{x} ; \mathrm{TSS}_{\mathrm{T}}=1200, \mathrm{ES}=2.5 \mathrm{x}, 3 \mathrm{x}\right)$ favor ratios heavily weighted towards having

326 more individuals versus more repeated measures (ratios ranging from approximately 5:1 to 10:1;

327 Figure 3B, C). Power to detect $\sigma^{2}{ }_{1 \mathrm{k}}$ is higher overall and less sensitive to deviations from the

328 optimum ratio than power to detect $\sigma^{2}{ }_{0 k}$ (Figure 3).

329 Power to detect $\sigma^{2}{ }_{v \mathrm{k}}$ follows a strikingly different pattern than $\sigma^{2}{ }_{0 \mathrm{k}}$ and $\sigma^{2}{ }_{\mathrm{lk}}$. Power to

330 detect $\sigma^{2}{ }_{v \mathrm{k}}$ is non-monotonic across ratios of individuals to the number of Bernoulli observations

331 within sampling occasions (Figure 4), and is an increasing function of the number of sampling

332 occasions (Figure 5C). At low sample sizes (e.g. $\mathrm{TSS}_{\mathrm{T}}=600$ ) power to detect $\sigma^{2}{ }_{v \mathrm{k}}$ is maximized

333 by devoting nearly all of the available resources to repeated measures within individuals (ratios

334 of approximately 1:30 to 3:40; Figure 4A); however, at a large sample size and effect size (e.g.

$\left.335 \mathrm{TSS}_{\mathrm{T}}=2,400, \mathrm{ES}=3.0\right)$ power is maximized at a ratio of individuals to Bernoulli observations

336 of approximately 5:6 (Figure 4C).

338 Scenario 2: Power under increasing non-target random effect variance

Power to detect differences in variance components is strongly affected by the proportion

340 of total variance that can be attributed to the target variance component (Figure 6). Increasing

341 variance in non-target random effects decreases power to detect differences in the target variance

342 parameter by treatment. However, the ratio of target to non-target variance does not alter the

343 optimal ratio of individuals to repeated measures for the target variance comparison (Figure 6). 
344 Panel A demonstrates that power to detect $\sigma^{2}{ }_{0 k}$ decreases substantially as the magnitude of

345 within-individual variation increases. Detecting differences in $\sigma^{2}{ }_{1 \mathrm{k}}$ depends only on total random

346 effect variation at extreme ratios of individuals to sampling occasions (e.g. 80:3) (Figure 6B).

347 Finally, detection of $\sigma_{v \mathrm{k}}^{2}$ is largely independent of the magnitude of among-individual variation

348 at large ratios of ES to non-target variance, as indicated by overlapping curves in Figure 6C.

349 However, when among-individual variation in intercept is very large (Figure 6C: Red curve),

350 power to detect $\sigma^{2}{ }_{v k}$ decreases because individual mean responses approach 0 or 1 , reducing the

351 amount of detectable within-individual variation.

352

353 Discussion

354 The power analyses presented here establish a framework for designing experiments

355 focused on detecting differences in variance components by treatment using GLMMs. These

356 results should serve as a baseline upon which researchers can expand to address their own

357 specific problems. Nevertheless, our findings reveal some important general trends that should

358 be considered when designing experiments. Our results demonstrate heterogeneity in power

359 across sampling schemes (ratio of individuals to repeated measures and partitioning of repeated

360 measures into sampling occasions and Bernoulli observations), and differences in which

361 sampling scheme maximizes power for different components of variance (Figures 2-5). As

362 expected, power declines rapidly for low sample sizes and small effect sizes (Figures 2-4).

363 However, for large $\mathrm{TSS}_{\mathrm{T}}$ and relatively large effect sizes (3 SD difference between treatments), >

$36480 \%$ power is retained across many different combinations of individuals to repeated measures

365 for each component of variance (Figures 2-5). Not surprisingly, power to detect differences in 
366 the target random effect declines with increasing variance in the non-target random effects 367 (Figure 6).

368 Power to detect $\sigma^{2}{ }_{0 \mathrm{k}}$ is non-monotonic across ratios of individuals to sampling occasions, 369 and is an increasing function of the number of Bernoulli observations per sampling occasion.

370 Power is maximized with ratios weighted towards having more individuals (Figure 2), and

371 quickly declines with alternative sampling ratios when total sample sizes and effect sizes are

372 small. The analyses are however more robust to deviations from this ratio when $\mathrm{TSS}_{\mathrm{T}}$ and ES are

373 large (Figure 2C). Finally, of all the random effect parameters we analyzed, power to detect $\sigma^{2} 0 \mathrm{k}$

374 is the most sensitive to the amount of "noise" present in the model, decreasing rapidly with

375 increasing within-individual variation (Figure 6).

376 Power to detect $\sigma^{2}{ }_{1 \mathrm{k}}$ is also non-monotonic across ratios of individuals to sampling

377 occasions, and is maximized with a ratio of individuals to sampling occasions ranging from 2:1

378 to 5:1 as $\mathrm{TSS}_{\mathrm{T}}$ increases (Figure 3). On average, testing for differences in $\sigma^{2}{ }_{1 \mathrm{k}}$ are more powerful 379 than for $\sigma_{0 \mathrm{k}}^{2}$ across all sampling schemes and ES (Figures 2, 3), and requires fewer samples to 380 obtain $80 \%$ power.

381 Finally, power to detect $\sigma^{2}{ }_{v \mathrm{k}}$ is non-monotonic across ratios of individuals to Bernoulli 382 observations and is an increasing function of the number of sampling occasions. Depending on 383 sample size, sampling schemes ranging from maximizing Bernoulli observations to ratios of 384 individuals to Bernoulli observations of 1:2 maximizes power (Figure 4). Unlike $\sigma^{2}{ }_{0 \mathrm{k}}$, power to 385 detect $\sigma^{2}{ }_{v \mathrm{k}}$ is largely independent of additional variance in the model (Figure 6C), such that 386 power to detect $\sigma^{2}{ }_{v \mathrm{k}}$ is nearly equivalent at all levels of $\sigma^{2}{ }_{0 \mathrm{k}}$ except under the case of extreme 387 values of $\sigma^{2} 0 \mathrm{k}$. 
Collectively these results indicate the importance of clearly defining a biological question, designating the focal random effect, and knowing the expected magnitude of total 390 variation when determining the appropriate experimental sampling design and $\mathrm{TSS}_{\mathrm{T}}$. Even at

391 larger effect sizes, failure to account for system noise can lead to insufficient power and a failed 392 experiment. Our findings should serve as a strong warning to empiricists interested in variance 393 components that power analyses should be performed when designing experiments in order to 394 overcome the problems of overall low power, large heterogeneity in power to detect different 395 variance components, and heterogeneity in sampling scheme required to optimize power. By introducing new strategies for analyzing variance among treatments we hope to inspire novel experimental designs in ecology and evolution. For example, the power analyses presented here can inform the design of experiments aimed at quantifying heterogeneous within-individual variation by environment, which may lead to novel insights on the adaptive significance of within-individual variation (Westneat, Wright \& Dingemanse, 2014). In addition, these analyses answer the calls of researchers over the last decade for methods to investigate effects of treatment level variance on the variance of dependent variables 403 (Benedetti-Cecchi, 2003). Transitions from one discrete environment to another (e.g. presence or absence of predators) are often classified as a form environmental variation, but switching between two distinct but relatively constant environments does not reflect environmental 406 variation per se, such as temporal changes in the magnitude, pattern, and/or frequency of the 407 environmental over time (Benedetti-Cecchi, 2003; Benedetti-Cecchi et al., 2006; Miner \& 408 Vonesh, 2004; Lawson et al., 2015). When this form of environmental variation is manipulated 409 or natural variation exploited in an experimental context, within-individual variation can be 410 described as the variable response of individuals to this variation in the environment. In this 
411 context, within-individual variation may itself be a form of phenotypic plasticity, and may have

412 profound implications for understanding the evolution of environmentally induced plasticity, and

413 the evolution of labile traits generally (Stamps, Briffa \& Biro, 2012; Biro \& Adriasenssens,

414 2013; Westneat, Wright \& Dingemanse, 2014).

415

416 Further Considerations

417 Heterogeneous within-individual variation

418 In our power analyses we have made a few important simplifying assumptions. First, we

419 assume that within-individual variation in both intercept and slope is homogenous among

420 individuals within the same treatment. Additionally, we assume homogeneity of within-

421 individual variance across an environmental gradient. However, these assumptions may not be

422 true for some natural or experimental populations. In fact, it has recently been proposed that

423 assessing the magnitude of variation in within-individual error variance within a single

424 individual across an environmental gradient or among individuals exposed to the same

425 environment/treatment is an important metric that may help to explain the evolution of plasticity

426 (Cleasby, Nakagawa \& Schielzeth, 2015; Westneat, Wright \& Dingemanse, 2014). Power to

427 detect differences in the magnitude of among-individual variation in within-individual variation

428 by treatment (Cleasby, Nakagawa \& Schielzeth, 2015) and heterogeneity of variance across an

429 environmental gradient are interesting research questions that deserve attention, but are beyond

430 the scope of this article. We also note that practicality limits exploration of increasingly

431 complicated scenarios, despite their conceivable statistical feasibility and intrinsic charm due to 432 complex novelty.

433 
434

435

436

437

438

439

440

441

442

443

444

445

446

447

448

449

450

451

452

453

454

455

456

Covariance among intercept, slope, and variance components

All of our simulations assessed power to detect differences in a single target variance comparison between treatments, holding all other variance parameters constant (Table S1).

However, manipulating non-target variation generates additional variation that is expected to decrease power to detect differences in the target variance parameter. Because we assumed no slope variation in models where intercepts were allowed to vary and no intercept variation in the models focused on variation in slopes, we did not discuss power to detect covariance terms. However, these parameters can co-vary and the covariation among these parameters may contain a wealth of biologically relevant information. For example, covariation between phenotypic plasticity and within-individual variation may be tightly linked via developmental tradeoffs, which can lead to greater developmental instability in highly plastic individuals (Tonsor, Elnaccash \& Scheiner, 2013). Indeed, it is not known whether an individual's reaction norm slope and within-individual variation around that reaction norm are always linked or if these relationships can be context-dependent. Similarly, we do not know if stronger behavioral responses lead to greater canalization of behavior. Understanding how to parameterize GLMM and how to optimize experiments to detect these covariances will be a useful step toward advancing evolutionary theory on adaptive, maladaptive and random patterns of variation. Covariance between intercept and slope has been described extensively in theoretical papers and has been explored in earlier power analyses for LMM (Dingemanse \& Dochtermann, 2013); however, empirical studies documenting significant covariance between these parameters remain rare (Mathot et al., 2011; Dingemanse et al., 2012). While covariance among these parameters may be uncommon, it is also likely that most experiments have insufficient power to detect such covariance. Additional analyses that determine power to detect significant 
457 differences in intercept and slope covariation for GLMMs is another important step considering

458 the lack of current evidence for covariation reported in the literature.

459

460 Within-individual variation in slope

461 Research, including ours, on among-individual variation in plasticity assumes fully

462 repeatable plasticity within each individual, causing among-individual differences in phenotypic 463 plasticity to be calculated using a single reaction norm for each individual (Dingemanse \& Wolf, 464 2013). However, quantifying only a single reaction norm for each individual fails to capture any 465 potential variation in plastic responses within an individual around its mean reaction norm, 466 which may inflate estimates of among-individual variation and mask important variation that is 467 subject to selection (Dingemanse \& Wolf, 2013). Despite the reasonable assumption that each 468 experimental individual would exhibit variation in their reaction norm if it were repeatedly 469 measured, we are aware of no studies that demonstrate repeatable behavioral plasticity for a 470 single individual when assessed multiple times.

472 Heterogeneity in sampling scheme and environment

473 In our simulations all individuals were measured an equal number of times and all 474 treatments contained the same number of individuals, a luxury often not available to empiricists 475 that often deal with missing data and unbalanced designs. Intuitively, unbalanced sampling 476 schemes will lower the power to detect among-individual variation (Van de Pol, 2012); however 477 we do not know the rate at which statistical power is lost with the magnitude of imbalance for a 478 particular sampling design. In highly unbalanced designs or when data have many missing 479 observations state-space models may be a more powerful alternative to GLMMs for separating 
480 different types of variability (Schnute, 1994). Future research should follow the lead of Van de

481 Pol, 2012 to determine how power to assess differences in variance for GLMM is affected by

482 incomplete sampling, specifically when only a single measure is available for some individuals.

483

484

Experiments with more than two treatments

485

Finally, these power analyses were created for a two-treatment scenario--"homogenous"

486

environmental variation treatment and a "variable" environmental variation treatment. However,

487

it is commonplace to have more than two treatments. Fortunately, our framework for conducting

488

power analyses can be easily generalized for exploring power for experiments with more than

489

two treatments (see supplemental material). In addition, syntax for the lme 4 package in R for

490

specifying GLMM is highly flexible and can be written to restrict variance components to be the

491

same in any number of treatments, while unique variance estimates can be obtained for any other

492

given treatment. For example in a four treatment experiment composed of four levels of predator

493

cue, two variance estimates could be obtained for among-individual variation (e.g. a single

494

estimate for the three treatments with the lowest levels of predator cue and one estimate for the

495

highest level of predator cue). As in the two-treatment scenario, differences in variance among

496

treatments in a multi-treatment scenario can be evaluated with a likelihood ratio test.

497

498 Conclusions

499

Despite the ubiquity of random intercepts and slopes GLMMs in ecology, evolution, and

500 behavior, the use of GLMMs to compare variance components among populations or among

501 experimental treatments is rare. We hope the power analyses presented here will spur novel

502 empirical research and assist readers in constructing appropriate experimental designs and 
503 statistical models to test how variance components are shaped by ecological and evolutionary

504 processes. We emphasize a clearly defined biological question and designation of the focal

505 random effect when designing experiments for this application due to unique ratios of

506 individuals to repeated measures required to optimize power for each variance parameter and

507 low overall power (in most cases $>1,000$ total Bernoulli observations per treatment needed to

508 achieve $80 \%$ power). Finally, we call for future work analyzing the accuracy and precision of

509 estimates comparing random effects by treatment for GLMMs (which our code facilitates)

510 similar to the work of Moineddin, Matheson \& Glazier, 2007 and Van de Pol, 2012 on the

511 accuracy and precision of random effects estimates. As Van de Pol points out, just because

512 power is high does not ensure the accuracy and precision of estimates. 


\section{Acknowledgements}

514 We thank the lab members of Dr. Michael McCoy's lab and Dr. Krista McCoy's lab for helpful 515 comments during the preparation of this manuscript. We also thank Daniel Hocking and one 516 anonymous reviewer for many helpful comments and suggestions that improved the clarity of 517 this manuscript.

\section{Data Accessibility}

519 R scripts are available in Supplement 1 


\section{References}

Bates, D., Maechler, M., Bolker, B., \& Walker S. 2014. Fitting linear mixed-effects models using lme4. arXiv reprint. arXiv: 1406.5823.

Bell, A.M., Hankison, S.J. \& Laskowski, K.L. 2009. The repeatability of behaviour: a metaanalysis. Animal Behaviour, 77, 771-783.

Benedetti-Cecchi, L. 2003. The importance of the variance around the mean effect size of ecological processes. Ecology, 84, 2335-2346.

Benedetti-Cecchi, L., Bertocci, I., Vaselli, S. \& Maggi, E. 2006. Temporal variance reverses the impact of high mean intensity of stress in climate change experiments. Ecology, 87, 2489-2499.

Biro, P.A. \& Adriaenssens, B. 2013. Predictability as a personality trait: consistent differences in intra-individual behavioral variation. The American Naturalist, 182, 621-629.

Bolker, B.M., Brooks, M.E., Clark, C.J., Geange, S.W., Poulsen, J.R., Stevens, M.H.H. \& White, J.S.S. 2009. Generalized linear mixed models: a practical guide for ecology and evolution. Trends in Ecology \& Evolution, 24, 127-135.

Bolker, B.M. 2015. Linear and generalized linear mixed models. Ecological Statistics: Contemporary theory and application (eds G.A. Fox, S. Negrete-Yankelevich \& V.J. Sosa), pp. 309-334. Oxford University Press, USA.

Browne, W.J., Subramanian, S.V., Jones, K. \& Goldstein, H. 2005. Variance partitioning in multilevel logistic models that exhibit overdispersion. Journal of the Royal Statistical Society Series A-Statistics in Society, 168, 599-613.

Cernicchiaro, N., Renter, D.G., Xiang, S., White, B.J. \& Bello, N.M. 2013. Hierarchical Bayesian modeling of heterogeneous variances in average daily weight gain of commercial feedlot cattle. Journal of animal science, 91, 2910-2919.

Cleasby, I.R., Nakagawa, S. \& Schielzeth, H. 2015. Quantifying the predictability of behaviour: statistical approaches for the study of between-individual variation in the within-individual variance. Methods in Ecology and Evolution, 6, 27-37.

De León, L.F., Raeymaekers, J.A., Bermingham, E., Podos, J., Herrel, A. \& Hendry, A. P. 2011. Exploring possible human influences on the evolution of Darwin's finches. Evolution, 65, $2258-2272$.

Dingemanse, N.J., Kazem, A.J.N., Réale, D. \& Wright, J. 2010. Behavioural reaction norms: animal personality meets individual plasticity. Trends in Ecology and Evolution, 25, 8189.

Dingemanse, N.J., Bouwman, K.M., van de Pol, M., van Overveld, T., Patrick, S.C., Matthysen, 

E. \& Quinn, J.L. 2012. Variation in personality and behavioural plasticity across four populations of the great tit Parus major. Journal of Animal Ecology, 81, 116-126.

564

589

590

591

592

593

594

595

Dingemanse N.J. \& Dochtermann N.A. 2013. Quantifying variation in behaviour: mixed-effect modeling approaches. Journal of Animal Ecology, 82, 39-54.

Dingemanse, N.J. \& Wolf, M. 2013. Between-individual differences in behavioural plasticity within populations: causes and consequences. Animal Behaviour, 85, 1031-1039.

Fitzmaurice, G.M., Laird, N.M. \& Ware, J.H. 2004. Applied Longitudinal Analysis, 2nd edn. Wiley, New Jersey, USA.

Gelman, A. \& Hill, J. 2006. Data Analysis Using Regression and Multi-level/Hierarchical Models. Cambridge University Press, England.

Ghalambor, C.K., Angeloni, L.M. \& Carroll, S.P. 2010. Behavior as phenotypic plasticity. Evolutionary behavioral ecology (eds D.F. Westneat \& C.W. Fox), pp. 90-197. Oxford University Press, USA.

Hill, W.G. \& Zhang, X.S. 2004. Effects on phenotypic variability of directional selection arising through genetic differences in residual variability. Genetical Research, 83, 121-132.

Hinde, J. 1982. Compound Poisson regression models. GLIM 82: Proceedings of the International Conference on Generalised Linear Models (ed R. Gilchrist), pp. 109-121. Springer, New York.

Jennions, M. \& Møller, A. 2003. A survey of the statistical power of research in behavioral ecology and animal behavior. Behavioral Ecology, 14, 438-445.

Johnson, P.C., Barry, S.J., Ferguson, H.M. \& Müller, P. 2014. Power analysis for generalized linear mixed models in ecology and evolution. Methods in Ecology and Evolution, 6, $133-142$.

Kizilkaya, K. \& Tempelman, R.J. 2005. A general approach to mixed effects modeling of residual variances in generalized linear mixed models. Genetics Selection Evolution, 37, $31-56$.

Lawson, C.R., Yngvild V., Bailey, L. \& van de Pol, M. 2015. Environmental variation and population responses to global change. Ecology Letters, doi: 10.1111/ele.12437

Lynch, M. \& Walsh, B. 1998. Genetics and Analysis of Quantitative Traits, 1st edn. Sinauer Press, Sunderland.

Martin, J.G.A. \& Réale, D. 2008. Temperament, risk assessment and habituation to novelty in eastern chipmunks, Tamias striatus. Animal Behaviour, 75, 309-318. 
598

599

600

601

602

603

604

605

606

607

608

609

610

611

612

613

614

615

616

617

618

619

620

621

622

623

624

625

626

627

628

629

630

631

632

633

634

635

636

637

638

639

640

Martin, J.G.A., Nussey, D.H., Wilson, A.J. \& Réale, D. 2011. Measuring individual differences in reaction norms in field and experimental studies: a power analysis of random regression models. Methods in Ecology and Evolution, 2, 362-374.

Mathot, K.J., van den Hout, P.J., Piersma, T., Kempenaers, B., Réale, D. \& Dingemanse, N.J. 2011. Disentangling the roles of frequency-vs. state-dependence in generating individual differences in behavioural plasticity. Ecology letters, 14, 1254-1262.

Miner, B.G. \& Vonesh, J.R. 2004. Effects of fine grain environmental variability on morphological plasticity. Ecology Letters, 7, 794-801.

Moineddin, R., Matheson, F.I. \& Glazier, R.H. 2007. A simulation study of sample size for multilevel logistic regression models. BMC Medical Research Methodology, 7, 1-34.

Moore, A.J., Brodie, E.D. III \& Wolf, J.B. 1997. Interacting phenotypes and the evolutionary process: I. Direct and indirect genetic effects of social interactions. Evolution, 51, 13521362.

Nakagawa, S. \& Schielzeth, H. 2010. Repeatability for Gaussian and non-Gaussian data: a practical guide for biologists. Biological Reviews, 85, 935-956.

Nussey, D.H., Wilson, A.J. \& Brommer, J.E. 2007. The evolutionary ecology of individual phenotypic plasticity in wild populations. Journal of Evolutionary Biology, 20, 831-844.

Pigliucci M. 2001. Phenotypic Plasticity: Beyond Nature and Nurture. John Hopkins University Press, Baltimore, MD.

Pinheiro, J. \& Bates, D. 2001. Mixed-Effects Models in S and S-PLUS. Springer, New York.

R Development Core Team. 2014. R: A Language and Environment for Statistical Computing. R Foundation for Statistical Computing, Vienna. URL http://www.R-project.org

Scherbaum, C.A. \& Ferreter, J.M. 2009. Estimating statistical power and required sample sizes for organizational research using multilevel modeling. Organizational Research Methods, $12,347-367$.

Schielzeth, H. \& Nakagawa, S. 2013. Nested by design: model fitting and interpretation in a mixed model era. Methods in Ecology and Evolution, 4, 14-24.

Schnute, J.T. 1994. A general framework for developing sequential fisheries models. Canadian Journal of Fisheries and Aquatic Sciences, 51, 1676-1688.

Stamps, J.A., Briffa, M. \& Biro, P.A. 2012. Unpredictable animals: individual differences in intraindividual variability (IIV). Animal Behaviour, 83, 1325-1334.

Stroup, W.W. 2012. Generalized linear mixed models: modern concepts, methods and applications. CRC Press, USA. 
641

642

643

644

645

646

647

648

649

650

651

652

653

654

655

656

657

658

659

660

661

662

663

664

665
Tonsor, S.J., Elnaccash, T.W. \& Scheiner, S.M. 2013. Developmental instability is genetically correlated with phenotypic plasticity, constraining heritability, and fitness. Evolution, 67, 2923-2935.

van de Pol, M. \& Wright, J. 2009. A simple method for distinguishing within-versus betweensubject effects using mixed models. Animal Behaviour, 77, 753-758.

Verbeke, G. \& Molenberghs, G. 2000. Linear Mixed Models for Longitudinal Data. Springer, New York.

Westneat, D.F., Hatch, M.I., Wetzel, D.P. \& Ensminger, A.L. 2011. Individual variation in parental care reaction norms: integration of personality and plasticity. The American Naturalist, 178, 652-667.

Westneat, D.F., Wright, J. \& Dingemanse, N.J. 2014. The biology hidden inside residual withinindividual phenotypic variation. Biological Reviews. doi:10. 1111/brv.12131

Wolf, M. \& Weissing, F.J. 2012. Animal personalities: consequences for ecology and evolution. Trends in Ecology \& Evolution, 27, 452-461.

Zuur, A.F., Leno, E.N., Walker, N., Saveliev, A.A. \& Smith, G.M. 2009. Mixed effects models and extensions in ecology with $R$. Springer, New York.

Zuur, A.F., Hilbe, J.M. \& Leno, E.N. 2013. A Beginner's Guide to GLM and GLMM with R: A Frequentist and Bayesian Perspective for Ecologists. Highland Statistics Ltd, Newburgh, UK. 


\section{Table $\mathbf{1}$ (on next page)}

Parameter values for all simulations

Table 1: For example, Scenario 1: Figure $2 \mathrm{C}$ illustrates power to detect differences in $\sigma^{2}{ }_{0 k}$ across ratios of individuals to sampling occasions with a TSS $_{T}$ of 2,400 at effect sizes of $2 x$, $2.5 \mathrm{x}$, and $3 \mathrm{x}$ difference in standard deviation by treatment. 


\begin{tabular}{|c|c|c|c|c|c|c|c|c|c|c|c|c|c|c|c|}
\hline $\begin{array}{c}\text { Target } \\
\text { Variance }\end{array}$ & \multicolumn{5}{|c|}{$\sigma_{0 \mathrm{k}}^{2}$} & \multicolumn{5}{|c|}{$\sigma^{2}{ }_{1 \mathrm{k}}$} & \multicolumn{5}{|c|}{$\sigma_{v \mathrm{k}}^{2}$} \\
\hline Scenario & & & 1 & & 2 & & the & & & 2 & & & 1 & & 2 \\
\hline Figure & $2 \mathrm{~A}$ & 2B & $2 \mathrm{C}$ & $5 \mathbf{A}$ & $6 A$ & 3A & 3B & $3 \mathrm{C}$ & 5B & $6 B$ & $4 \mathrm{~A}$ & 4B & $4 \mathrm{C}$ & $5 \mathrm{C}$ & $6 \mathrm{C}$ \\
\hline Parameter & \multicolumn{3}{|c|}{ Sampling Occasions } & $\begin{array}{l}\text { Bernoulli } \\
\text { Obs }\end{array}$ & $\sigma^{2}{ }_{v \mathrm{k}}$ & \multicolumn{3}{|c|}{ Sampling Occasions } & $\begin{array}{c}\text { Bernoulli } \\
\text { Obs }\end{array}$ & $\sigma^{2}{ }_{v \mathrm{k}}$ & \multicolumn{3}{|c|}{ Bernoulli Observations } & $\begin{array}{c}\text { Sampling } \\
\text { Occasions }\end{array}$ & $\sigma_{0 \mathrm{k}}^{2}$ \\
\hline $\mathbf{T S S}_{\mathrm{T}}$ & 600 & 1,200 & 2,400 & $240-3,600$ & 2,400 & 300 & 600 & 1,200 & $120-1,800$ & 1,200 & 600 & 1,200 & 2,400 & $240-3,600$ & 2,400 \\
\hline \# Individuals & $2-60$ & $2-120$ & $2-240$ & $120-2$ & $2-240$ & $2-30$ & $2-60$ & $2-120$ & $60-2$ & $2-120$ & $2-60$ & $2-120$ & $2-240$ & $2-120$ & $2-240$ \\
\hline $\begin{array}{l}\text { \# Sampling } \\
\text { Occasions }\end{array}$ & $60-2$ & $120-2$ & $240-2$ & $2-120$ & $240-2$ & $30-2$ & $60-2$ & $120-2$ & $2-60$ & $120-2$ & 5 & 5 & 5 & $1-15$ & 5 \\
\hline $\begin{array}{c}\text { \# Bernoulli } \\
\text { Observations }\end{array}$ & 5 & 5 & 5 & $1-15$ & 5 & 5 & 5 & 5 & $1-15$ & 5 & $60-2$ & $120-2$ & $240-2$ & $120-2$ & $240-2$ \\
\hline Effect Sizes & $2 ; 2.5 ; 3$ & $2 ; 2.5 ; 3$ & $2 ; 2.5 ; 3$ & 2.5 & 2.5 & $2 ; 2.5 ; 3$ & $2 ; 2.5 ; 3$ & $2 ; 2.5 ; 3$ & 2.5 & 2.5 & $2 ; 2.5 ; 3$ & $2 ; 2.5 ; 3$ & $2 ; 2.5 ; 3$ & 2.5 & 2.5 \\
\hline
\end{tabular}


Figure 1 (on next page)

Reaction norm plots for a two treatment LMM

Figure 1: In all graphs bolded black lines depict treatment mean reaction norms and thin lines depict reaction norms of individuals. Grey envelopes in (C) illustrate the magnitude of within-individual intercept variation. Here among-individual variation in intercept (A), slope (B), and within-individual variation in intercept (C) is larger in treatment 2. 


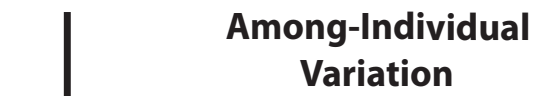

(A)

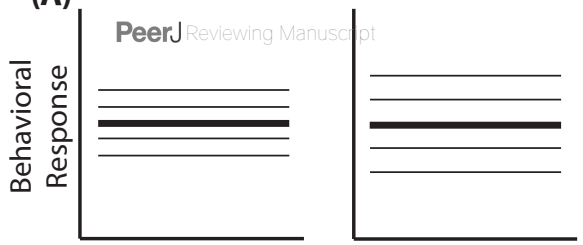

(B)

$\frac{0}{\text { 음 }}$
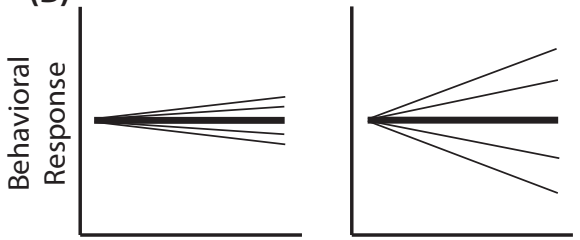

Within-Individual

Variation

(C)

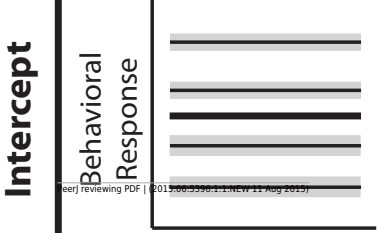

Environment

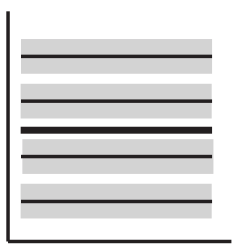

Environment 
Figure 2 (on next page)

Power to detect differences by treatment in among-indiviudal variation in intercept

Figure 2: Power to detect differences in $\sigma_{0 k}$ for three effect sizes (ratio of $\sigma_{0 k}$ between treatments) and three $\mathrm{TSS}_{\mathrm{T}}$ (total sample size per treatment). Colored circles indicate the ratio of individuals to sampling occasions that optimizes power for each effect size. Each scenario was simulated with 5 Bernoulli observations per sampling occasion. 


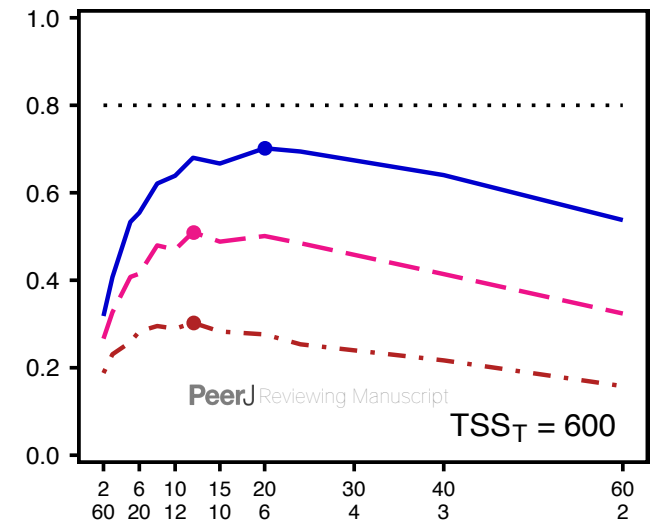

(B)
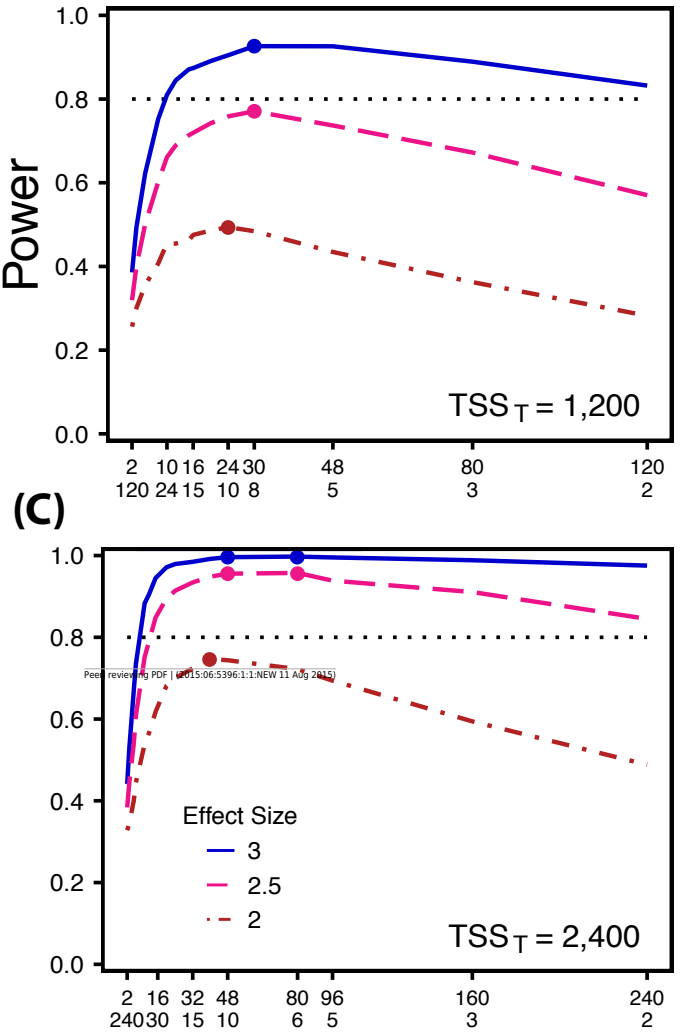

Individuals Sampling Occasions 
Figure 3 (on next page)

Power to detect differences by treatment in among-indiviudal variation in slope

Figure 3: Power to detect differences in $\sigma_{1 k}$ for three effect sizes and three TSS $_{\mathrm{T}}$. Colored circles indicate the ratio of individuals to sampling occasions that optimizes power for each effect size. Each scenario was simulated with 5 Bernoulli observations per sampling occasion. 


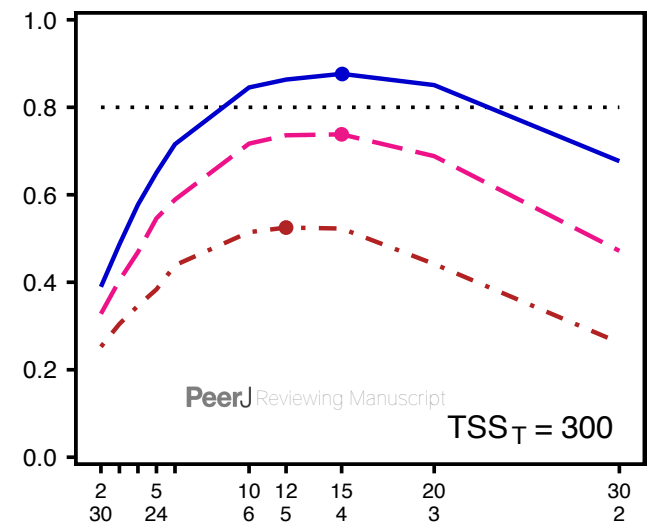

(B)

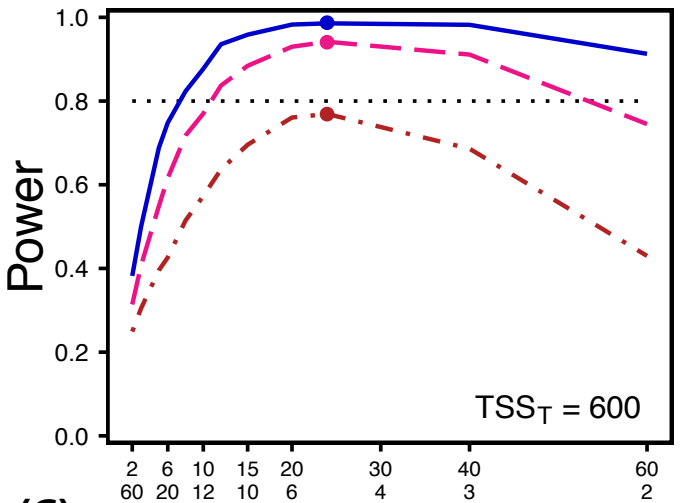

(C)

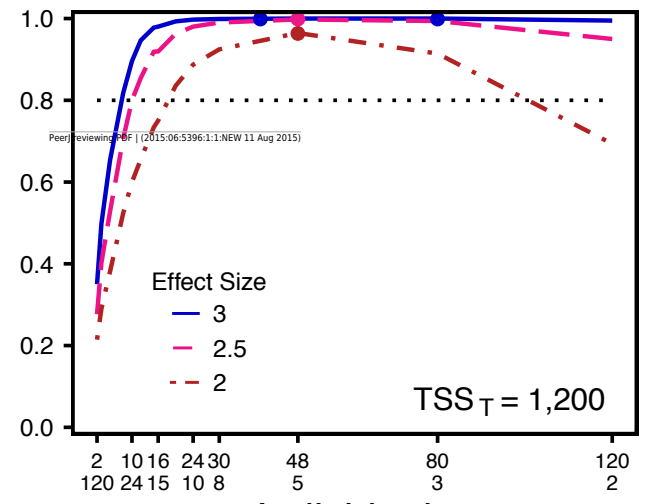

Individuals Sampling Occasions 
Figure 4 (on next page)

Power to detect differences by treatment in within-indiviudal variation in intercept

Figure 4: Power to detect differences in $\sigma_{v k}$ for three effect sizes and three TSS $_{T}$. Colored circles indicate the ratio of individuals to Bernoulli observations that optimizes power for each effect size. Each scenario was simulated with 5 sampling occasions. 


\section{$\sigma_{\mathrm{vk}}$}

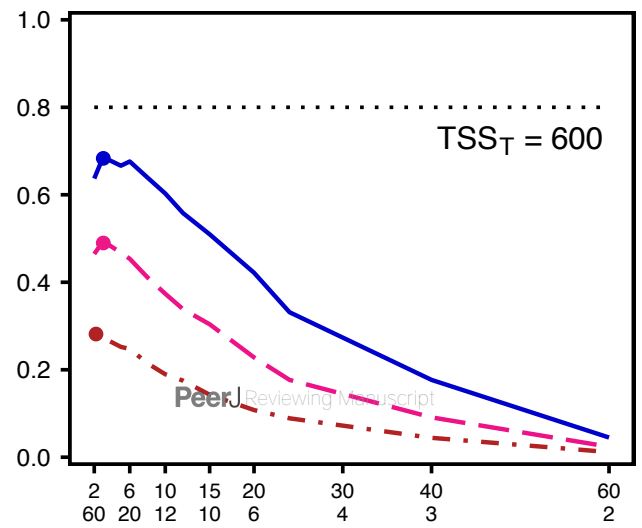

(B)
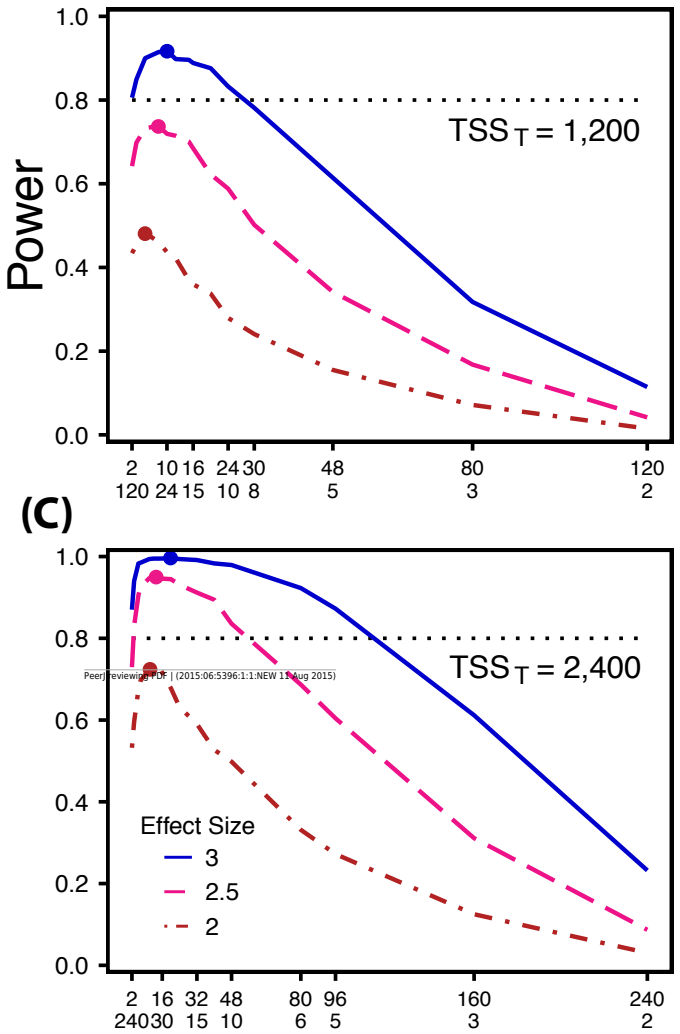

Individuals

Bernoulli Observations 
Figure 5 (on next page)

Power under increasing Bernoulli observations or sampling occasions

Figure 5: Power to detect differences in $\sigma_{0 k}(A)$ and $\sigma_{1 k}(B)$ under increasing Bernoulli observations per sampling occasion; $\sigma_{v k}(C)$ under increasing sampling occasions. In (A) and (B) ratios of individuals to sampling occasions follow figures $2 B$ and $3 B$ respectively. In (C) ratios of individuals to Bernoulli observations follows figure $4 \mathrm{~B}$. In (A) and (B) colored circles indicate the ratio of individuals to sampling occasions that optimizes power for each level of Bernoulli observations. In (C) colored circles indicate the ratio of individuals to Bernoulli observations that optimizes power for each level of sampling occasions. 
Figure 6 (on next page)

Power under increasing non-target variation

Figure 6: Power to detect differences in $\sigma_{0 k}(A)$ and $\sigma_{1 k}(B)$ under increasing variation in $\sigma_{v k}$; $\sigma_{v k}(C)$ under increasing variation in $\sigma_{0 k}$. Noise is given as the ratio of effect size to variation in the non-target variance parameter. In (A) and (B) ratios of individuals to sampling occasions follow figures $2 \mathrm{C}$ and $3 \mathrm{C}$ respectively. In $(\mathrm{C})$ ratios of individuals to Bernoulli observations follows figure $4 \mathrm{C}$. Colored circles indicate the ratio of individuals to sampling occasions (A, B) or Bernoulli observations (C) that optimizes power for each level of noise. 
